\title{
TESTING LMC MICROLENSING SCENARIOS: THE DISCRIMINATION POWER OF THE SuperMACHO MICROLENSING SURVEY
}

\author{
A. Rest, ${ }^{1,2}$ C. Stubbs, ${ }^{3}$ A. C. Becker, G. A. Miknaitis, A. Miceli, R. Covarrubias, and S. L. Hawley \\ Department of Astronomy, University of Washington, Box 351580, Seattle, WA 98195 \\ R. C. Smith, N. B. Suntzeff, K. Olsen, J. L. Prieto, and R. Hiriart \\ Cerro Tololo Inter-American Observatory, Casilla 603, La Serena, Chile \\ D. L. WELCH \\ Department of Physics and Astronomy, McMaster University, Hamilton, ON L8S 4M1, Canada
}

K. H. Cook, S. Nikolaev, M. Huber, and G. Prochtor ${ }^{4}$

Lawrence Livermore National Laboratory, 7000 East Avenue, Livermore, CA 94550

A. Clocchiatti ${ }^{5}$ and D. Minniti ${ }^{6}$

Department of Astronomy, Pontificia Universidad Católica de Chile, Casilla 306, Santiago 22, Chile

A. Garg and P. Challis ${ }^{7}$

Physics Department, Harvard University, 17 Oxford Street, Cambridge, MA 02138

AND

S. C. Keller and B. P. Schmidt

Research School of Astronomy and Astrophysics, Australian National University, Weston, ACT 2611, Australia

Received 2004 May 21; accepted 2005 August 4

\begin{abstract}
Characterizing the nature and spatial distribution of the lensing objects that produce the previously measured microlensing optical depth toward the Large Magellanic Cloud (LMC) remains an open problem. We present an appraisal of the ability of the SuperMACHO Project, a next-generation microlensing survey directed toward the LMC, to discriminate between various proposed lensing populations. We consider two scenarios: lensing by a uniform foreground screen of objects and self-lensing by LMC stars. The optical depth for "screen lensing" is essentially constant across the face of the LMC, whereas the optical depth for self-lensing shows a strong spatial dependence. We have carried out extensive simulations, based on data obtained during the first year of the project, to assess the SuperMACHO survey's ability to discriminate between these two scenarios. In our simulations we predict the expected number of observed microlensing events for various LMC models for each of our fields by adding artificial stars to the images and estimating the spatial and temporal efficiency of detecting microlensing events using Monte Carlo methods. We find that the event rate itself shows significant sensitivity to the choice of the LMC luminosity function, limiting the conclusions that can be drawn from the absolute rate. If instead we determine the differential event rate across the LMC, we will decrease the impact of these systematic biases and render our conclusions more robust. With this approach the SuperMACHO Project should be able to distinguish between the two categories of lens populations. This will provide important constraints on the nature of the lensing objects and their contributions to the Galactic dark matter halo.
\end{abstract}

Subject headings: dark matter — galaxies: halos — galaxies: structure — Galaxy: structure gravitational lensing - Magellanic Clouds

Online material: color figures

\section{INTRODUCTION}

An elegant way to further our understanding of dark matter halos and to search for astrophysical dark matter candidates is to utilize the defining feature of the dark matter: the effect of its gravitational field. Paczyński (1986) suggested searching for dark matter in the form of MAssive Compact Halo Objects

\footnotetext{
${ }^{1}$ Now at Cerro Tololo Inter-American Observatory (CTIO), La Serena, Chile. $\mathrm{CTIO}$ is a division of the National Optical Astronomy Observatory (NOAO).

${ }^{2}$ Goldberg Fellow.

3 Now at Departments of Astronomy and of Physics, Harvard University, Cambridge, MA 02138.

${ }^{4}$ Now at Astronomy Department, University of California, Santa Cruz, CA 95064.

5 Supported by FONDECYT grant 1000524.

${ }^{6}$ Supported by Fondap Center for Astrophysics grant 15010003.

7 Harvard-Smithsonian Center for Astrophysics, 60 Garden Street, Cambridge, MA 02138.
}

(MACHOs) using gravitational microlensing. Several groups followed this suggestion and established microlensing searches toward the Large Magellanic Cloud (LMC) and other nearby galaxies. The MACHO group reported 13-17 microlensing events toward the LMC (Alcock et al. 2000), with event timescales ranging between 34 and 230 days. They estimated the microlensing optical depth toward the LMC to be $\tau=1.2_{-0.3}^{+0.4} \times 10^{-7}$. If we assume that MACHOs are responsible for this optical depth, then a typical halo model allows for a MACHO halo fraction of $20 \%(95 \%$ confidence interval of $8 \%-50 \%)$, with MACHO masses ranging between 0.15 and $0.9 M_{\odot}$. The OGLE (Optical Gravitational Lensing Experiment) collaboration also reported one LMC microlensing event (Udalski et al. 1997). Notably, none of the surveys toward the LMC have detected events with timescales $1 \mathrm{hr} \leq \hat{t} \leq 10$ days. This lack of short-timescale events puts a strong upper limit on the abundance of low-mass dark matter objects: objects with masses $10^{-7} M_{\odot}<m<10^{-3} M_{\odot}$ 
make up less than $25 \%$ of the halo dark matter. Further, less than $10 \%$ of a standard spherical halo is made of MACHOs in the $3.5 \times 10^{-7} M_{\odot}<m<4.5 \times 10^{-5} M_{\odot}$ mass range (Alcock et al. 1998). It is interesting to note that early results from surveys toward M31 are confirming a nonnegligible MACHO content in M31's halo (Uglesich et al. 2004; Calchi Novati et al. 2005; Jetzer et al. 2004).

Recent data and publications have reinvigorated discussion concerning microlensing rates toward the LMC. The EROS-2 project has found evidence for variability in an event classified as microlensing by the MACHO Project (Tisserand \& Milsztajn 2005) while also reporting four new microlensing candidates toward the LMC. In addition, using neural networks to analyze a subset of MACHO light curves including all events classified as microlensing by MACHO, Evans \& Belokurov (2004) find an optical depth toward the LMC more consistent with that expected from known stellar populations. Bennett et al. (2005), however, show that previously unpublished data support the microlensing interpretation questioned by Evans \& Belokurov. Accounting for new evidence that removes some MACHO events from the microlensing set, Bennett (2005) recalculated the microlensing optical depth toward the LMC using efficiencies determined for the entire data set. His revised optical depth is $\tau=(1.0 \pm 0.3) \times 10^{-7}$. He found the MACHO data to be consistent with a $\sim 16 \%$ MACHO halo. EROS-2 calculates a preliminary optical depth of about $1.5 \times 10^{-8}$ (Jetzer et al. 2004).

Despite these varying constraints on the MACHO halo fraction, the microlensing event rate (reported by Alcock et al. 2000) toward the LMC significantly exceeds that expected from known visible components of our Galaxy. This raises the question of where the lenses reside. Unfortunately, the main observable in any given microlensing event, its duration, depends on a combination of lens mass, position, and velocity relative to the source. Any conclusion about the spatial location of the lens population therefore depends on the assumptions made about its mass and velocity. We note that in cases where the light curve exhibits a departure from the point-source/point-lens event shape (due to a binary lens, a binary source, noninertial motion in the lensing system, etc.), this degeneracy can be lifted.

Using the standard Galactic and LMC model, an optical depth of about $10^{-8}$ is expected toward the LMC from known Galaxy (e.g., thick-disk, halo) and LMC components. This is significantly lower than the optical depth of $1.2 \times 10^{-7}$ detected by the MACHO survey (Alcock et al. 2000). Given the difficulty associated with locating the lenses along the line of sight, previous microlensing surveys of the LMC have been unable to discriminate between a variety of possible sources for the excess LMC event rate. These include (1) lensing by a population of MACHOs in the Galactic halo, (2) lensing by a previously undetected thick-disk component of our Galaxy, (3) disk-bar or barbar self-lensing of the LMC, or (4) lensing by an intervening dwarf galaxy or tidal tail.

See $\S 2$ for a more detailed discussion of these populations. Due to the limited number of events observed to date, it is not yet clear which scenario or combination of scenarios explains the observed lensing signal.

The SuperMACHO Project is an ongoing 5 year microlensing survey of the LMC that is being carried out with the specific goal of answering the question, "Where do the lenses responsible for the measured event rates toward the LMC reside?" (Stubbs 1999).

We have designed our survey to provide a significant increase in the number of detected events. This will allow a more robust assessment of the spatial variation of the microlensing optical depth across the face of the LMC and will clarify whether the observed optical depth can be accounted for by LMC self-lensing, the most popular alternative to lensing by MACHOs. This paper presents an appraisal of SuperMACHO's ability to accomplish this goal. This assessment is based on extensive simulations that use observational data obtained during the first observing season. The LMC luminosity function (LF) plays a prominent role in this calculation, and we present an extensive analysis of this in a future paper (A. Rest et al. 2006, in preparation).

\subsection{A First Step: Foreground Lenses or LMC Lenses?}

As a first step toward determining the nature of the lensing population, we consider and evaluate two lensing scenarios:

1. Screen lensing.-Lensing caused by a uniform (on the angular scale of the LMC) foreground lensing population. Examples are lensing by the Galactic halo or thick disk or by a many-degree scale intervening population of lenses.

2. LMC self-lensing.-Lensing where the lens population is either the same as the source population or spatially close to the source population. Examples are LMC disk-disk, disk-bar, and bar-bar lensing and lensing of the LMC disk by a tidal tail within the LMC.

We consider it a sensible first step to ascertain the extent to which these may be responsible for the microlensing events seen toward the LMC.

The lensing rate for self-lensing shows a strong spatial dependence (e.g., the lensing rate for LMC bar-bar lensing is proportional to $N_{\text {bar }}^{2}$, where $N$ is the areal density of stars), whereas the lensing rate of screen lensing is directly proportional to the number of source stars observed. The goal of the SuperMACHO Project is to determine which or what mixture of these two categories causes the observed microlensing rate. One key ingredient to achieving this is to increase the number of detected events. We do this in two ways: (1) increasing the number of source stars monitored and (2) increasing our event detection efficiencies by performing difference image analysis. The corresponding improvement in event detection rate should move us out of the realm of small-number statistics and allow us to determine the spatial distribution of the events on the sky (see Fig. 1). This, in turn, should allow us to discriminate between the two possibilities described above.

Our approach in assessing the survey's discrimination capability is to

1. Use actual LMC images obtained with the survey instrumentation to obtain star count information for each of our fields.

2. Add simulated microlensed flux to the frames and assess the survey's event detection efficiency for each field as a function of input event parameters.

3. Estimate, for the observed LMC optical depth, the likely event distribution statistics across the different fields under different lensing scenarios.

4. Given the anticipated event detection rates, devise statistics that maximize the survey's ability to discriminate between screen lensing and LMC self-lensing.

5. Assess the SuperMACHO survey's sensitivity to a specific illustrative LMC self-lensing scenario, namely, the displaced LMC bar model proposed by Zhao \& Evans (2000).

These steps are laid out below. In $\S 3$ we describe the SuperMACHO survey strategy and the image analysis pipeline of the project. Section 4 summarizes our parameterization of the LMC stellar LF, an essential ingredient needed to go from the 


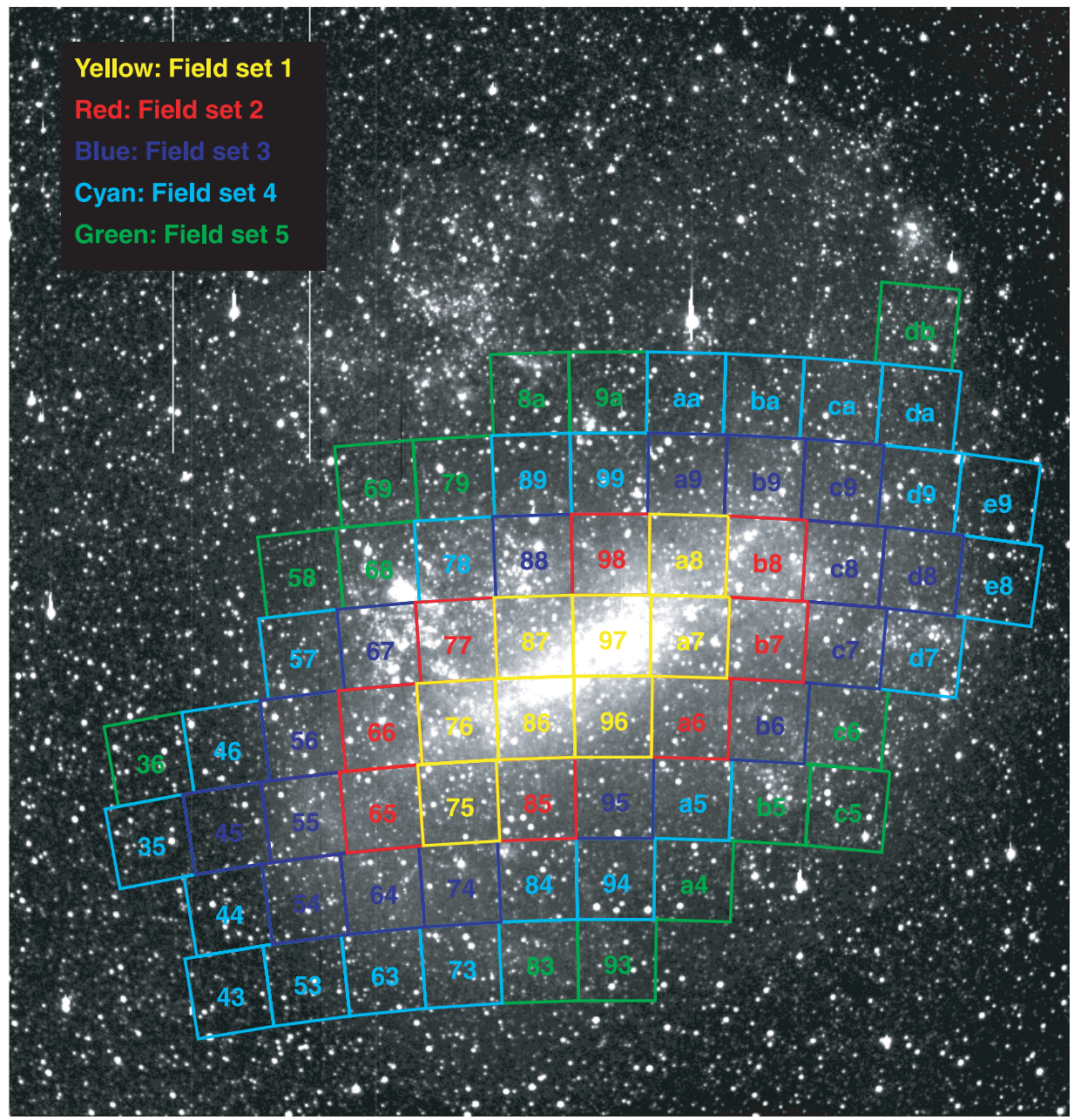

FIG. 1. - SuperMACHO fields superposed onto the LMC. The fields are divided into sets 1-5, based on their respective star density determined with the Zhao \& Evans (2000) LMC bar model. Set 1 is the most crowded (yellow) and set 5 is the least crowded set (green). (LMC image courtesy of G. Bothun.)

observable quantity, the number of microlensing events detected, to the physical properties of interest, in particular, the mass and spatial distributions of the lens population. We present detailed LF analysis in a companion paper (A. Rest et al. 2006, in preparation). In $\S 5$ we then use these results to model the number of detected microlensing events for the different candidate populations, and we predict whether it will be possible for SuperMACHO to distinguish between screen lensing and self-lensing. Before we begin a detailed examination of SuperMACHO's discrimination capability, we begin by examining the various lensing scenarios in greater detail.

\section{LENS POPULATION CANDIDATES}

The known and expected components of the Galactic/LMC system each contribute to the event rate toward the LMC. In the following we discuss the different lens population candidates, each of which is problematic in some respect.

\subsection{Milky Way Halo Lenses?}

If the lenses reside as MACHOs in the Galactic halo, their inferred typical mass is $0.1-1 M_{\odot}$ (Alcock et al. 2000). If we assume that such a lens population is composed of some known astronomical object, the most likely candidates are white dwarfs (WDs). There are some indications that there might be a previously undetected population of old WDs in the Galaxy (Ibata et al. 1999, 2000; Méndez \& Minniti 2000; Oppenheimer et al. 2001; Nelson et al. 2002), favoring this interpretation. However, Kilic et al. (2004) have shown that some of these WD candidates are background AGNs. Like any scenario populating the Galactic halo with stellar remnants, the halo WD explanation is challenged by stellar formation and evolution theory: the stellar progenitors are expected to enrich the gas and/or stars to a greater degree than has been observed. In addition, if other galaxies have similar halos, then their progenitor populations should be observable in galaxies at high redshift. There are ways out of these constraints, e.g., by assuming nonstandard initial mass functions (Chabrier et al. 1996; Chabrier 1999), or by anticipating lower metal yields from old, low-metallicity main-sequence progenitors, or perhaps by allowing that the processed ejecta remain in the form of hot gas as yet undetected (Fields et al. 2000). All these attempts require fine-tuning of the models or invoke unlikely physics, rendering them somewhat unsatisfying. The interpretation that the detected faint WDs are members of the Galactic halo is certainly not uncontested (e.g., see Richer 2003; Crézé et al. 2004).

\subsection{Thick-Disk Lenses?}

An alternate interpretation is that the lenses belong kinematically to the thick disk (Reid et al. 2001). In this scenario, the inferred number of WDs exceeds the number expected from the 
known thick disk or spheroid, forcing the invocation of an undetected very thick disk as an alternative to a halo population of lenses (Gates et al. 1998). Gyuk \& Gates (1999) showed that such disks may be able to reproduce the observed optical depth toward the LMC. More recently, they showed that the predicted properties of such a population are consistent with the observed properties of the WDs (Gates \& Gyuk 2001). Since the total mass of such thick-disk WDs needed to account for the observed optical depth seen toward the LMC is much smaller than the total mass needed in the halo, this explanation solves some of the stellar evolution and chemical enrichment problems. Only more detailed observations can determine to which populations the WDs might belong or if they reside in a new, unknown component of the Galaxy.

\subsection{LMC Self-lensing?}

A third possible interpretation of the optical depth toward the LMC is that the lenses are not part of our Galaxy but rather of the LMC itself, i.e., lens and source population reside within the LMC. This was first suggested independently by Sahu (1994) and $\mathrm{Wu}$ (1994) and is denoted as LMC self-lensing. The most common self-lensing scenarios invoke pairs of LMC bar, disk, and halo objects as source and/or lens populations. Several groups find self-lensing optical depths close to $10^{-7}$ and claim that therefore the observed optical depth can be explained with self-lensing (e.g., Aubourg et al. 1999; Zhao \& Evans 2000). That claim has been disputed by several other groups (e.g., Sahu 1994; Gould 1995; Alcock et al. 1997; Gyuk et al. 2000; Mancini et al. 2004) who find optical depths closer to $10^{-8}$ and thus not sufficient to explain the observed optical depth. The main differences between the different estimates of the optical depth come from different choices of LMC models and model parameters (Gyuk et al. 2000). Recent observations indicate that there might be kinematic (carbon star sample, Graff et al. 2000; RR Lyrae stars, Minniti et al. 2003) and photometric subcomponents of the LMC (Weinberg \& Nikolaev 2001; van der Marel \& Cioni 2001; van der Marel 2001). The unvirialized subcomponents can be caused by the tidal interaction between the LMC, the Galaxy, and/or the SMC. Some theoretical models that invoke such unvirialized subcomponents find that the optical depth is significantly increased and may account for half or even all of the microlensing event rate (Graff et al. 2000; Zhao \& Evans 2000). The predicted events show peculiarities in their photometric, kinematic, and spatial distributions that can be used to distinguish between LMC stars and the other lens population candidates. For example, one of the two near-clump MACHO events (LMC-1) is a few tenths of a magnitude fainter than the clump, and Zhao et al. (2000) argue that this is suggestive of having the lensed source star in a distinct population spatially separated and behind the LMC (and possibly more reddened). Using Hubble Space Telescope (HST) observations, Alcock et al. (2001a) do not find any significant evidence for such systematically redder source stars. Their results marginally favor halo lensing. In addition, recent observations do not show any significant signs of kinematic outliers in the LMC (Zhao et al. 2003), restricting any additional kinematically distinct population to less than the $1 \%$ of the LMC stars.

\subsection{Galactic Halo Substructure?}

There has been increasing evidence that the Galactic stellar halo is not smooth. Beside the Magellanic Stream, another full-fledged tidal stream, the Sagittarius dwarf galaxy, which currently is passing through the Galactic disk, has been detected (Ibata et al. 1995,
2001; Yanny et al. 2000; Ivezić et al. 2000; Vivas et al. 2001). There is also tentative evidence for other tidal streams in the Galactic halo (Newberg et al. 2002) and between the Galaxy and the LMC (Zaritsky \& Lin 1997). Such an intervening dwarf galaxy or tidal tails could also cause the high microlensing event rate (Zaritsky \& Lin 1997; Zhao 1998; Weinberg \& Nikolaev 2001).

\section{OBSERVATIONS: THE IMPLEMENTATION OF THE SuperMACHO SURVEY}

The primary motivation for starting the SuperMACHO survey was the collection of a sufficient number of microlensing events toward the LMC so that a statistical analysis can be made of competing theories for the location of the lenses. Previous LMC microlensing surveys such as MACHO and EROS have shown that microlensing can be detected and characterized with photometric sampling every few days and that LMC microlensing events are not shorter than 2 weeks. These surveys also highlighted the benefits of good seeing to reduce the effects of blending of source stars, while the simultaneous collection of data in multiple passbands to assess the achromaticity of candidate events has not proven very useful due to the effects of blending. With these lessons in mind, the SuperMACHO Project was proposed to use a larger aperture to detect fainter events, at a better seeing site, in a single filter, and fit into the restrictions of using a nondedicated telescope by observing every second night. The image analysis was designed to use difference image photometry, which had been shown by previous surveys to be more efficient in detecting microlensing. The SuperMACHO proposal ${ }^{8}$ was allocated 150 halfnights, distributed over 5 years, on the Cerro Tololo Inter-American Observatory (CTIO) Blanco $4 \mathrm{~m}$ telescope through the NOAO Survey Program. The survey started in 2001 and will run through 2005 . We note that we have waived any proprietary data access rights and that the SuperMACHO survey images are accessible through the NOAO Science Archive on the NOAO Web site. 9

Observations are carried out every other night in dark time during the months of October, November, and December, when the LMC is most accessible from CTIO. We use the $8 \mathrm{~K} \times 8 \mathrm{~K}$ MOSAIC II CCD imager with a field of view of $0.33 \mathrm{deg}^{2}$. The eight SITe $2 \mathrm{~K} \times 4 \mathrm{~K}$ CCDs are read out in dual-amplifier mode (i.e., different halves of each CCD are read out in parallel through separate amplifiers) to increase our observing efficiency. In order to maximize the throughput we use a custom-made broadband filter ( $V R$ filter) from 500 to $750 \mathrm{~nm}$. The atmospheric dispersion corrector on the MOSAIC II imager allows for the use of this broad band without a commensurate point-spread function (PSF) degradation.

In devising an observing strategy we want to find a good balance between maximizing the number of events detected and assuring a uniform spatial coverage. The work described here has guided our decisions on how to best spend the telescope time on the sky. We have defined a grid of 68 fields over the face of the LMC. Previous microlensing surveys found that the distribution of microlensing event durations ${ }^{10}$ toward the LMC has its peak at about $\hat{t}=80$ days, with virtually no event lasting less than 2 weeks. In order to sample the light curves adequately and sufficiently, we observe all fields every other night during dark time. This also serves to equalize, to first order, the event detection efficiency due to sampling effects across the fields. There remains the field-dependent detection efficiency due to the different stellar densities and due to intentional inequality in exposure times.

\footnotetext{
${ }^{8}$ See http://www.ctio.noao.edu/supermacho.

9 See ftp://archive.tuc.noao.edu/pub.

${ }^{10}$ The event duration is twice the Einstein radius crossing time.
} 
The distribution of the available observing time in a half-night across the LMC is driven by two conflicting considerations: the need to maximize the number of stars that we monitor and the need to survey as large a region as possible in order to discriminate between the different candidate lens populations. If the only goal were to maximize the number of monitored stars and, consequently, the number of detected microlensing events, we would concentrate on deep exposures of the central region of the LMC. Maximizing the number of microlensing events is, however, less important than achieving maximum discrimination between models.

We have adopted the strategy outlined by Gould (1999) to achieve a distribution of exposure times that maximizes the microlensing event rate subject to the constraints of a given spatial sampling and the differing sensitivity between the inner and outer regions of the LMC. The basic idea is that the distribution of exposure times for a microlensing survey is optimized when a shift of $\delta t$ in exposure from field $\mathrm{A}$ to field $\mathrm{B}$ gains as many stars in B as are lost in field A. At this extremum, $\delta N_{A} / \delta t=\delta N_{B} / \delta t$. This condition must be achieved subject to two constraints. First, the total exposure time plus the total time spent on readout must equal the number of workable hours in a halfnight. Second, the number of sources monitored in the inner and outer regions of the LMC must be balanced to achieve the desired spatial coverage.

We have used the stellar density normalizations described in $\S 4$ and a simple division into inner and outer fields to optimize the distribution of exposure times given the properties of the MOSAIC II imager on the CTIO $4 \mathrm{~m}$ telescope. We have set a minimum exposure time of $25 \mathrm{~s}$ in order to assure coverage of the sparser fields and a maximum exposure time of $200 \mathrm{~s}$ in order to avoid saturation effects.

The SuperMACHO Project started observations in 2001 September. The data analysis pipeline is currently implemented as a combination of C code, IRAF, Perl, and Python scripting tied together to provide an integrated but modular environment (Smith et al. 2002).

The first steps of the data processing, cross talk correction and astrometric calibration, are best done on the whole image because the units are not completely independent. The rest of the image reduction, as well as all of the transient analysis, breaks down naturally into 16 independent units, the amplifier images, ${ }^{11}$ and can therefore be efficiently handled in parallel. We employ a cluster of 18 CPUs with a 6.5 terabyte redundant disk array.

Standard photometry of transient or variable objects becomes inefficient in highly crowded images; therefore, we use a method called difference image analysis, which has rapidly evolved in the last few years. The first implementation was by Phillips \& Davis (1995), who introduced a method that registered images, matched the PSF, and matched the flux of objects in order to detect transients. Derivatives of difference image analysis have been widely applied in various projects (e.g., MACHO, Alcock et al. 1999a; M31 microlensing, Crotts et al. 1999; OGLE, Woźniak 2000; WeCAPP, Gössl \& Riffeser 2002; the Deep Lens Survey, Becker et al. 2004). Since the PSF varies over the field of view due to optical distortions or out-of-focus images, for example, it is essential to use a spatially varying kernel (Alard \& Lupton 1998; Alard 2000).

One of the main problems with the image-differencing approach is that there are more residuals, e.g., cosmic rays and bleeds, than genuinely variable objects in the difference image. Therefore, standard profile-fitting software like DoPHOT (Schechter et al.

\footnotetext{
11 The MOSAIC II has eight CCDs. Each CCD is read out by two amplifiers.
}

1993) has problems determining the proper PSF used to perform photometry in the difference image. When the difference image is analyzed with our customized version of DoPHOT, we force the PSF to be the one determined for the original, flattened image. Applying this a priori knowledge of the PSF helps to guard against bright false positives, such as cosmic rays and noise peaks, which generally do not have a stellar PSF.

All detections are added into a database. Once the database is loaded and objects have been identified, queries are performed on new objects that are then classified. Objects of interest are then passed to a graphical user interface displaying stamps from the image, template, and difference image for visual classification and interpretation.

\section{LMC LUMINOSITY FUNCTIONS}

In order to determine the optical depth from the observable quantities (the number and duration of detected events), the number of potential source stars must be known. This depends on both exposure depth and on the LF of the source-star population. Knowing the LMC LF is essential for the analysis (and prediction) of microlensing event rates.

For this analysis we require the true LF for the stellar population of the whole LMC. The MACHO survey used HST images (Alcock et al. 1999b, 2001b) to determine the LF down to $V \sim 24$ for selected bar fields. They found that for all fields the LF was well fit with a power law with identical slope for $V \lesssim 22.5$, leveling out for fainter magnitudes. Even the HST LF shows a spread at magnitudes fainter than 22.5 (see Figs. 2 and 3 of Alcock et al. 2001b). Since our survey is most sensitive to microlensing events with source-star magnitudes in the range of 22-25 (see Fig. 6, top), these differences are important. Instead of relying on a single LF for our analysis, we decided to explore a variety of LFs so that we can quantify the impact of choosing an incorrect LF on our conclusions. We used five different LFs to represent the possible range of LFs in the LMC or to represent possible variations in the global LF. Two of these LFs are the "limiting cases," while the three intermediate LFs are based on either LFs from the solar neighborhood or direct fits to our LMC photometry. As explained below, all five LFs are tied to a single-power-law fit to the bright end of the LF, and the faint end spans a plausible range of luminosity distributions. We present our detailed analysis of the LMC LF in a companion paper (A. Rest et al. 2006, in preparation) and summarize its results here.

We divided each of the 68 fields that we observe into 16 subfields based on the area covered by the MOSAIC II amplifiers (see Fig. 1). For each of these subfields, we determined an independent LF. First, we fit a single power law with slope $\beta$ and stellar density parameter $\Phi_{0}$ (stars per square arcminute) to the bright end of the LF with a superposed Gaussian function representing the red clump:

$\Phi^{*}(M)=\Phi_{0} 10^{\beta M}+\frac{N_{\mathrm{RC}}}{\sigma_{\mathrm{RC}} \sqrt{2 \pi}} \exp \left[-\left(\frac{M_{\mathrm{RC}}-M}{2 \sigma_{\mathrm{RC}}}\right)^{2}\right]$,

where $N_{\mathrm{RC}}, M_{\mathrm{RC}}$, and $\sigma_{\mathrm{RC}}$ are the total number, the average magnitude, and the spread of red clump stars, respectively. The instrumental magnitude $M$ is defined as

$$
M=-2.5 \log f
$$

where $f$ is the flux in counts. Figure 2 shows the observed LF (circles) for the magnitude range in which the completeness is close to $100 \%$ for amplifier 4 of field sme9. The dotted line 


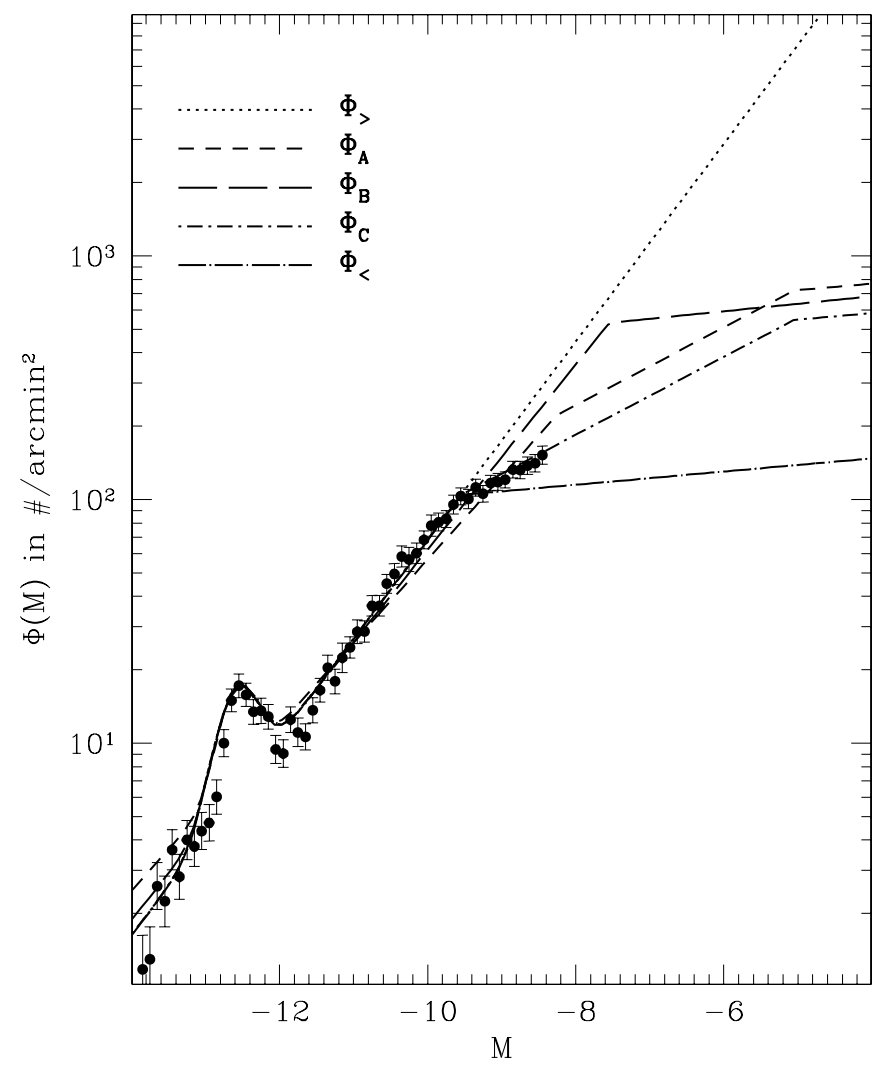

FIG. 2.-Different LFs $\Phi_{>}, \Phi_{A}, \Phi_{B}, \Phi_{C}$, and $\Phi_{<}$for amplifier 4 of field sme9 vs. the instrumental magnitude $M$. The units on the $y$-axis are stars per square arcminute and magnitude bin. The dotted line is the bright-end LF function parameterized as a single power law $\left(\Phi_{>}\right)$fitted to the completeness-corrected observed LF (filled circles) at the bright end. [See the electronic edition of the Journal for a color version of this figure.]

(denoted as $\Phi_{>}$) is a completeness-corrected fit of $\Phi^{*}(M)$ to the data in the magnitude range $M_{\mathrm{RC}}-2.5 \leq M \leq M_{\mathrm{RC}}+2.5$. For fainter magnitudes $(M \gtrsim-9.5)$ the fit clearly overestimates the number of observed stars. This break in power law is well known and documented for the solar neighborhood (Kroupa et al. 1993; Reid \& Hawley 2000; Chabrier 2001), and it is also seen in the LMC (Holtzman et al. 1997; Hunter et al. 1997; Olsen 1999; Alcock et al. 2001b). Thus, fitting a single power law $\Phi_{>}$to the bright end of the LFs sets the stellar density parameter for each subfield and is the upper bound to plausible LFs.

Since the faint end deviates noticeably from the single power law, we parameterize the LFs as a combination of three power laws:

$$
\Phi(M)= \begin{cases}\Phi_{1} 10^{\beta_{1} M} & M<M_{1}, \\ \Phi_{1} 10^{\left(\beta_{1}-\beta_{2}\right) M_{1}} 10^{\beta_{2} M} & M_{1} \leq M<M_{2}, \\ \Phi_{1} 10^{\left(\beta_{1}-\beta_{2}\right) M_{1}+\left(\beta_{2}-\beta_{3}\right) M_{2}} 10^{\beta_{3} M} & M \geq M_{2},\end{cases}
$$

where $M$ is defined in equation (2). Some of our candidate LFs are only single or double power laws. In these cases we disregard the functions that do not apply. We use the fitted red clump peak magnitude as the anchor point for the break magnitudes; i.e., $M_{1}$ and $M_{2}$ are always with respect to $M_{\mathrm{RC}}$. LFs:

The following is a description of how we construct the five

1. Upper limit $L F\left(\Phi_{>}\right)$.- The upper limit of the LF (Fig. 2, dotted line) is set by assuming that the power-law $\Phi^{*}(M)$ (see eq. [1]) fitted to the bright end continues to the faint end without a break $\left(\Phi_{1} \equiv \Phi_{0}, \beta_{1}=\beta\right.$ for all $\left.M\right)$.

2. Local neighborhood $L F\left(\Phi_{A}\right)$ semiempirical mass-luminosity relation.-Reid et al. (2002) estimate a three-power-law function as one of the possibilities of the present-day mass function using a semiempirical mass-luminosity relation. We convert this present-day mass function back into a LF using the massluminosity function for lower (Delfosse et al. 2000; Reid et al. 2002) and brighter (Reid et al. 2002) main-sequence stars, giving $\beta_{1}=0.34, \beta_{2}=0.16$, and $\beta_{3}=0.27$ with the two break magnitudes at $V=3.91$ and $V=7.11$. Using an unreddened absolute $V$ magnitude of the red clump peak of 0.39 (K. Olsen 2005 , private communication), we can express the break magnitudes relative to the red clump as $M_{1}=M_{\mathrm{RC}}+4.3$ and $M_{2}=M_{\mathrm{RC}}+$ 7.5. For each subfield we determine the stellar density parameter $\Phi_{A 0}$ and the red clump peak magnitude $M_{\mathrm{RC}}$ by fitting the singlepower-law $\Phi^{*}(M)$ (see eq. [1]) to the bright end of the LF with a fixed slope of $\beta=\beta_{1}=0.34$. This LF is shown as the shortdashed line in Figure 2.

3. Local neighborhood LF $\left(\Phi_{B}\right)$ empirical mass-luminosity relation.-Another proposed form of the present-day mass function by Reid et al. (2002) is a double power law based on an empirical mass-luminosity function. In the same way as for $\Phi_{A}$, we estimate $\beta_{1}=0.38, \beta_{2}=0.0314$, and $M_{1}=M_{\mathrm{RC}}+5.0$. The stellar density parameter $\Phi_{B 0}$ is determined by fitting the singlepower-law $\Phi^{*}(M)$ to the bright end of the LF with a fixed slope of $\beta=\beta_{1}=0.38$. This LF is shown as the long-dashed line in Figure 2.

4. Empirical LF $\left(\Phi_{C}\right)$.-As our empirical LF $\Phi_{C}$, we fit the single-power-law $\Phi^{*}(M)$ to the bright end of the LF, yielding $\Phi_{1} \equiv \Phi_{0}$ and $\beta_{1}=\beta$. We fit a second power law to the faint end of one of our sparse subfields, where the break in power law is virtually unaffected by completeness (see Fig. 2), yielding $M_{1}=$ $M_{\mathrm{RC}}+3.0$ and $\beta_{2}=0.16$. Note that the break 3 magnitudes fainter than the red clump peak magnitude is in very good agreement with the break seen in HST images of the LMC (see Alcock et al. 2001b). Since we do not have data going deep enough to see the second break, we assume the same break magnitude and slope for the third power law, $M_{2}=M_{\mathrm{RC}}+7.5$ and $\beta_{3}=0.027$, respectively, as determined for $\Phi_{A}$. This LF is shown as the shortdash-dotted line in Figure 2.

5. Lower limit $L F\left(\Phi_{<}\right)$.- - Similar to $\Phi_{>}$and $\Phi_{C}$, we determine $\Phi_{1}$ and $\beta_{1}$ by fitting the single-power-law $\Phi^{*}(M)$ to the bright end of the LF. As the first break in power law, we use the same break we found for $\Phi_{C}: M_{1}=M_{\mathrm{RC}}+3.0$. As the slope we choose the smallest slope of any of the power laws, $\beta_{2}=0.027$. This choice underestimates the LF at the faint end. This LF is shown as the long-dash-dotted line in Figure 2.

TABLE 1

Luminosity Function Parameterization

\begin{tabular}{ccccccc}
\hline \hline $\begin{array}{l}\mathrm{LF} \\
(1)\end{array}$ & $\begin{array}{l}\Phi_{1} \\
(2)\end{array}$ & $\begin{array}{c}\beta_{1} \\
(3)\end{array}$ & $\begin{array}{c}\beta_{2} \\
(4)\end{array}$ & $\begin{array}{c}\beta_{3} \\
(5)\end{array}$ & $\begin{array}{c}M_{1} \\
(6)\end{array}$ & $\begin{array}{c}M_{2} \\
(7)\end{array}$ \\
\hline$\Phi_{>} \ldots \ldots \ldots \ldots \ldots$. & $\Phi_{0}$ & $\beta$ & $\ldots$ & $\ldots$ & $\ldots$ & $\ldots$ \\
$\Phi_{A} \ldots \ldots \ldots \ldots \ldots$. & $\Phi_{A 0}$ & 0.34 & 0.16 & 0.027 & $M_{\mathrm{RC}}+4.3$ & $M_{\mathrm{RC}}+7.5$ \\
$\Phi_{B} \ldots \ldots \ldots \ldots \ldots$. & $\Phi_{B 0}$ & 0.38 & 0.031 & $\ldots$ & $M_{\mathrm{RC}}+5.0$ & $\ldots$ \\
$\Phi_{C \ldots \ldots \ldots \ldots \ldots .} \ldots \ldots \ldots \ldots$ & $\Phi_{0}$ & $\beta$ & 0.16 & 0.027 & $M_{\mathrm{RC}}+3.0$ & $M_{\mathrm{RC}}+7.5$ \\
$\Phi_{<\ldots \ldots \ldots \ldots \ldots .}$ & $\Phi_{0}$ & $\beta$ & 0.027 & $\ldots$ & $M_{\mathrm{RC}}+3.0$ & $\ldots$ \\
\hline
\end{tabular}

Notes.-Overview of the different LF parameterizations used for computing the number of monitored sources, in the terminology used in the text. Col. (1): Name of the LF model. Col. (2): Stellar density parameter used. Cols. (3)-(5): Relevant power-law slopes, if applicable. Cols. (6) and (7): Transition magnitude between power laws with respect to the fitted red clump magnitude $M_{\mathrm{RC}}$. 
An overview of the different LFs is given in Table 1. In a future paper (A. Rest et al. 2006, in preparation) we will present a more detailed description on how we derive the LFs.

We use these different trial LFs in the following section, where we predict event rates in different microlensing scenarios. We show that a differential rate analysis of the data can discriminate between models independently of the actual underlying LF.

\section{EVENT-RATE PREDICTION}

The SuperMACHO Project's initial goal is to distinguish between two broad categories of lensing: screen lensing and selflensing. We use data from the first year to predict the number of detected microlensing events from the different candidate populations in order to test whether the SuperMACHO Project is able to distinguish between them. We add artificial stars to the images and determine the completeness of the detections. With that, we estimate the spatial and temporal efficiency of detecting microlensing events in our data sets. In combination with the stellar LFs described above, we predict and compare the number of microlensing events for various screen-lensing and selflensing scenarios.

The number of observed events depends on both the source and lens populations. In general, estimating the number of detectable microlensing events $N_{\mathrm{ml}}$ is complicated, as it requires detailed knowledge about number density, velocity distribution, and other properties of the population. A good approximation is given by

$$
N_{\mathrm{ml}}=N_{\mathrm{obs}} \tau \mathcal{E}_{t}
$$

where $N_{\text {obs }}$ is the number of monitored stars for which microlensing can be detected, $\tau$ is the microlensing optical depth, and $\mathcal{E}_{t}$ is the sampling efficiency. This approximation separates the photometric and temporal completeness, which makes the calculation much simpler. The number of monitored stars contains the photometric completeness and is

$$
N_{\mathrm{obs}}=\int \Phi(M) \mathcal{E}(M) d M,
$$

where $\mathcal{E}(M)$ is the efficiency of detecting microlensing for a star with magnitude $M$, and $\Phi(M)$ is the LF. The temporal completeness is contained in $\mathcal{E}_{t}$ as

$$
\mathcal{E}_{t}=\left[\int\left(\frac{\hat{t}}{T}\right) \frac{D(\hat{t})}{P_{T}(\hat{t})} d \hat{t}\right]^{-1},
$$

where $T$ is the effective survey duration, $\hat{t}$ is the duration of a microlensing event, $P_{T}(\hat{t})$ is the probability that the event is detected within $T$ given the temporal cadence of the survey, and $D(\hat{t})$ is a normalized distribution of $\hat{t}$.

We calculate $N_{\text {obs }}$ and $\mathcal{E}_{t}$ independently for each field and amplifier, as described below.

\subsection{Number of Observed Stars}

The best way to estimate the number of observed stars is to perform Monte Carlo simulations with all the images used. The shortcoming of this method is that it is very CPU-intensive and terabytes of images have to be simultaneously available on disk. Our goal is to predict a lower limit on the number of events; therefore, we choose a slightly different approach and perform Monte Carlo simulations on only a subset of images subject to certain assumptions.
The question we have chosen to answer is, what is the probability $\mathcal{E}(M)$ that the flux of a star with a given magnitude $M$ is magnified during a microlensing event by a detectable amount? Let us assume that a microlensing event is detectable if the difference flux at peak has a signal-to-noise ratio $S / N \geq 5$. Whether such an event is then indeed detected depends on the temporal cadence as well as the seeing and transparency of the observing nights. We fold this into the temporal completeness analysis in $\S 5.2$.

During a microlensing event, the source star flux $f_{0}$ gets amplified to $f=f_{0} A$ with an amplification that can be expressed as $A(u)=\left(u^{2}+2\right) /\left[u\left(u^{2}+4\right)^{1 / 2}\right]$, where $u$ is the angular separation between source and lens in units of the Einstein angle. With difference imaging, the quantity we measure is not the total amplified flux, but the difference in flux, $\Delta f=f-f_{0}$, between the amplified and unamplified source star. By measuring only the difference flux, we avoid confusion due to crowding and blended sources. Using the difference images, then, we construct light curves of the difference flux, $\Delta f$.

Using equation (2), we can then express this flux difference as an instrumental magnitude $M_{\text {diff }}$, where

$$
\begin{aligned}
M_{\text {diff }}(M, u) & =-2.5 \log (\Delta f) \\
& =M-2.5 \log [A(u)-1] .
\end{aligned}
$$

We note that this is the instrumental magnitude of the flux difference, which is not the difference in magnitude between the amplified and unamplified source star.

In $\S 6$ we show that nearly all of the expected microlensing events have source stars in the magnitude range of $22.3<M_{V R}<$ 25.3. Thus we can assume that the unamplified source-star flux does not contribute significantly to the noise in the photometry, i.e., that the photometry is background noise dominated. Within this limit, then, instead of adding the unamplified flux $f_{0}$ to the template image, and the amplified flux $f$ to the image, we can just add the flux difference $\Delta f$ to the detection image to test whether we can detect such a flux difference. For this simulation we add flux into the images only and process these images through our difference image pipeline in the standard way. Because there is no source flux in the template, we measure the added difference flux, $\Delta f$, in the resultant difference image. We then derive what fraction of the artificial stars we recover with a $\mathrm{S} / \mathrm{N} \geq 5$ to obtain the empirical completeness function $C\left(M_{\text {diff }}\right)$. This allows us to estimate the probability that a microlensed star with intrinsic magnitude $M$ has a change in flux whose $\mathrm{S} / \mathrm{N}$ is $\geq 5$ at maximum amplification:

$$
\mathcal{E}(M)=\int C\left[M_{\mathrm{diff}}\left(M, u_{0}\right)\right] d u_{0}
$$

where $u_{0}$ is the angular separation at maximum brightness. With equation (5), the number of observed stars $N_{\text {obs }}$ can then be calculated using the LF $\Phi(M)$ for a given field and amplifier (see $\S 4)$.

\subsection{Sampling Efficiency}

The temporal cadence and observing conditions such as seeing and transparency have a significant impact on event detection efficiency. This is folded into $P(\hat{t})$ and thus implicitly into $\mathcal{E}_{t}$ (see eq. [6]).

In order to estimate $P(\hat{t})$ we perform Monte Carlo simulations. During each year microlensing events are drawn at random to have peak amplification sometime during an interval of $T=300$ days. 
This interval is dictated by the extent of the period of actual observing (100 days) and the need to ensure that real events that reach peak amplification before or after that interval (but are observable during the survey months) are represented in the simulation. The 100 day padding at both ends of the observation period is driven by the characteristic $\hat{t}$ found by the MACHO Project. The SuperMACHO observation periods are usually allocated in three runs of about 10 nights of bi-nightly observing separated by 2 weeks of bright time. Factors such as weather, instrument failures, and computer down time are taken into account by randomly eliminating one out of four nights. For a given $\hat{t}$, we realize 1000 light curves for each microlensing impact parameter $0<u_{0}<0.5$ in increments of 0.0125 . By choosing $u_{0}=0.5$ as our upper limit we ensure that we have at least a magnification by a factor of 2.18 . This will allow us to eliminate astrophysical sources of low-amplitude variability. Because the optical depth is typically calculated for $u_{0} \leq 1$, this simulation will only recover $50 \%$ of the number of events usually associated with a given optical depth. The time of maximum is randomly chosen within $T$. As a lower limit, we assume that all microlensing events observed have a difference flux with $\mathrm{S} / \mathrm{N}\left(W_{0}, t_{0}\right)=5$, where $W_{0}$ is the FWHM of the seeing at time $t_{0}$ of maximum amplification. Then the $\mathrm{S} / \mathrm{N}$ of a detection on another night at time $t$ can be estimated as

$$
\mathrm{S} / \mathrm{N}(W, t)=\left(\frac{W_{0}}{W}\right)^{2} \frac{A(t)-1}{A_{\max }-1} \mathrm{~S} / \mathrm{N}\left(W_{0}, t_{0}\right) .
$$

We randomly draw $W_{0}$ and $W$ from the distribution of seeing in the first year of the survey (see Fig. 3). We want to emphasize that this is a conservative lower limit, since the stars we count in $N_{\text {obs }}$ have a $\mathrm{S} / \mathrm{N} \geq 5$ in the difference flux at maximum amplification, whereas our Monte Carlo simulations assume $\mathrm{S} / \mathrm{N}=5$ at peak.

For each light curve we estimate the $\mathrm{S} / \mathrm{N}$ for every night we have taken data based on the FWHM and amplification. The decision whether we do or do not detect such a light curve is based on the following additional criteria:

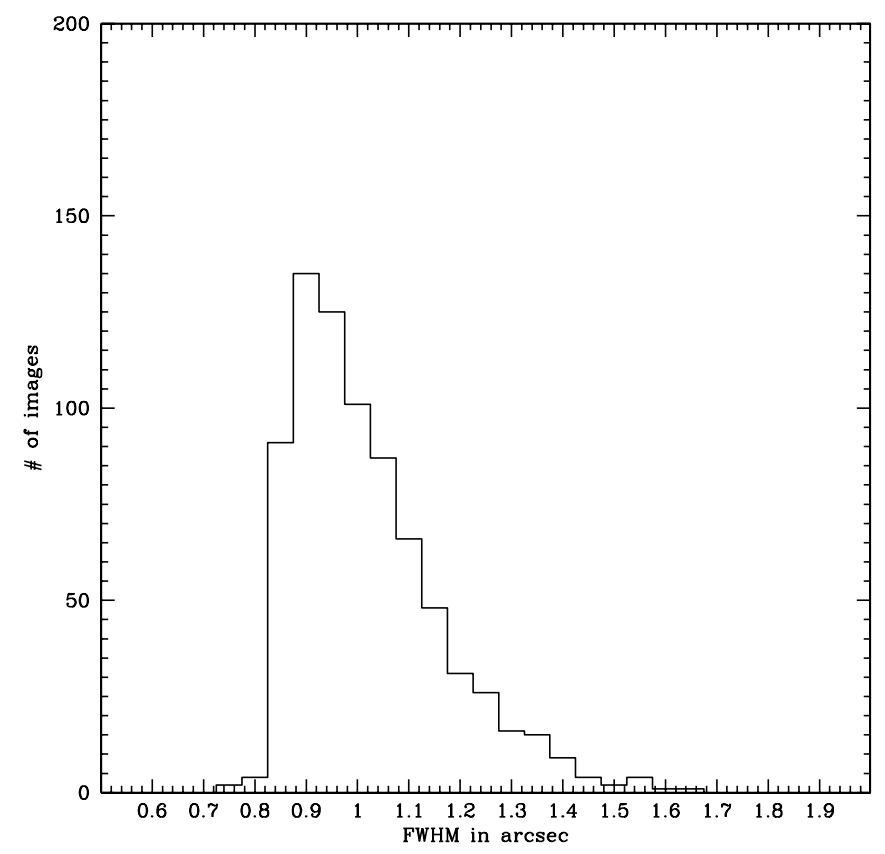

Fig. 3.- Seeing (FWHM) histogram for the 2001/2002 run for amplifier 4. The average is 1.202 with a standard deviation of 0.14 .

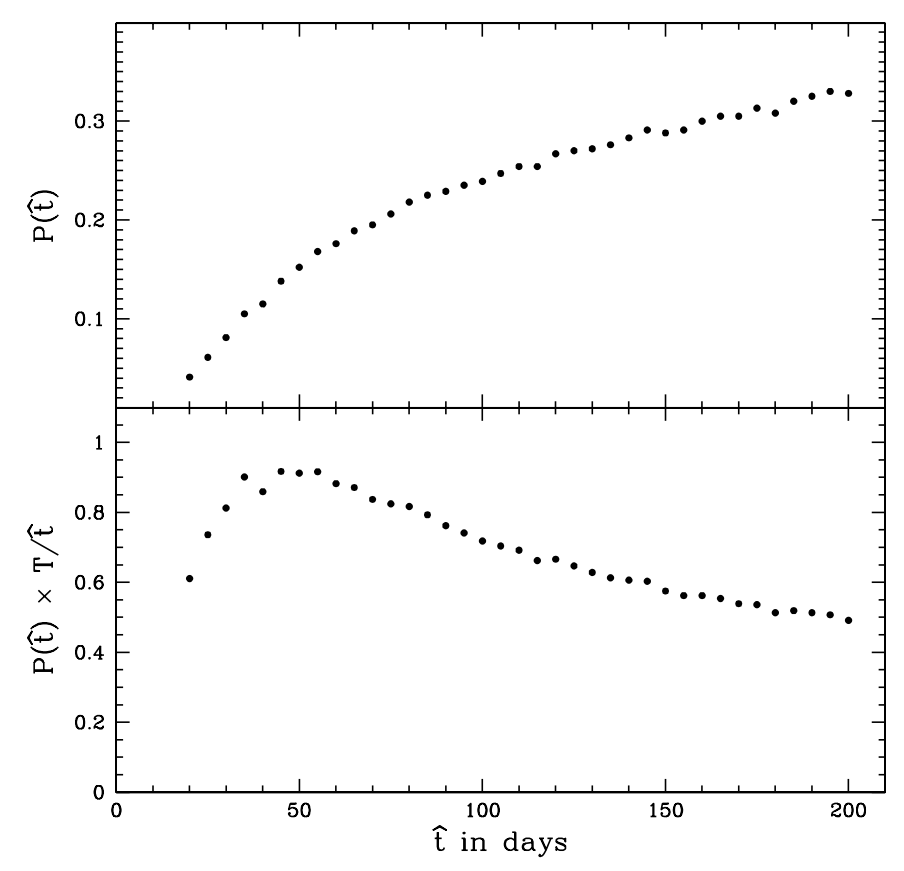

FIG. 4.-Probability of detecting a microlensing event with event duration of $\hat{t}$. The top panel shows the probability $P(\hat{t})$ of detecting a microlensing event with event duration of $\hat{t}$. The bottom panel shows the more intuitive measure $P(\hat{t}) \times(T / \hat{t})$, which is the number of microlensing events detected per $1 / \tau$ stars during an interval $T$, assuming that all microlensing events have an event duration of $\hat{t}$. The decrease of detection probability for decreasing event duration is countered by that fact that for shorter event durations more microlensing events happen per $1 / \tau$ stars in the given observing time. This causes the peak at $\hat{t} \sim 50$ days.

1. At least two detections on the rising arm of the light curve with a $\mathrm{S} / \mathrm{N}>2.0$.

2. At least four detections have a $\mathrm{S} / \mathrm{N}>2.0$.

This ensures that the events are "contained" within the survey coverage time and that there are enough significant detections to "trigger" an alert for follow-up observations. These cuts define the upper limit on the number of microlensing events we recover. We note the above set of criteria are insufficient to discriminate between microlensing and the population of background events in the actual survey. They are, however, comparable to the trigger criteria used in the MACHO alert system. Here we only consider the event sample in the case where there is no need for further discrimination. Discriminating microlensing is a separate problem and beyond the scope of this paper.

In the final analysis, the initial cuts described above will define a population of candidate events. We will apply additional cuts to eliminate contaminants such as supernovae, AGNs, and intrinsically variable stars. We will use photometric and spectroscopic follow-up observations to help define this advanced set of cuts. We note that these cuts will lower our detection efficiencies and require a reanalysis when they are determined.

The top panel of Figure 4 shows the probability $P(\hat{t})$ of detecting a microlensing event with event duration of $\hat{t}$. This event detection probability increases with event duration. Note that the probability is well below 1.0. This is because the interval used to define the input microlensing population was $T=300$ days, much longer than the actual annual observing duration ( $\sim 100$ days), and the probability of detecting an event with a peak time $t_{0}$ well outside the time the observations are taken is rather small. This effectively cancels out later on since we multiply by $T$ when calculating $\mathcal{E}_{t}$ (see eq. [6]).

A more intuitive measure is $P(\hat{t}) \times(T / \hat{t})$, which is the number of microlensing events detected per $1 / \tau$ stars during $T$ assuming 
that all microlensing events have an event duration of $\hat{t}$ (see Fig. 4, bottom). The decrease of detection probability for decreasing event duration is countered by that fact that for smaller event durations more microlensing events happen per $1 / \tau$ stars in the given observing time. This causes the peak at $\hat{t} \sim 50$ days.

Existing microlensing event statistics suggest that the event duration has a peak at about 80 days. Therefore we choose as $D(\hat{t})$ a Gaussian distribution with the peak at 80 days and with a spread of 20 days as inferred from Figure 9 in Alcock et al. (2000). Using equation (6), we can now evaluate

$$
\mathcal{E}_{t} \sim 0.8 .
$$

Thus we will observe about 0.8 microlensing events per $1 / \tau$ observed stars in one survey year (spanning 100 days).

\subsection{Bar-Disk Self-lensing Models for the LMC}

The optical depth from self-lensing remains a matter of controversy. Some studies find rather small values in the range of $(1.0-8.0) \times 10^{-8}$ (e.g., Alcock et al. 1997; Gyuk et al. 2000; Jetzer et al. 2002; Mancini et al. 2004), whereas other studies suggest optical depths up to $1.5 \times 10^{-7}$ (e.g., Zhao \& Evans 2000). This controversy arises from the still rather imprecise knowledge of the structure of the LMC and consequent differences in the adopted models.

The Zhao \& Evans (2000) models derived in their paper are concrete and testable self-lensing models that we will use in the following sections to predict the SuperMACHO self-lensing event rate. As pointed out above, these models, denoted as model set A (see $\S 5.3 .1$ ), predict a rather large optical depth and are thus more favorable to a self-lensing interpretation of LMC microlensing. We then improve upon their models (see $\S$ 5.3.2) for an alternative, more realistic model set, denoted as model set $\mathrm{B}$. This allows us to make a direct spatial comparison between self-lensing and screen-lensing event rates.

\subsubsection{Zhao \& Evans (2000) Model Set A}

Zhao \& Evans (2000) derive in their paper concrete and testable self-lensing models. They define a coordinate system with $X, Y$, and $Z$ being decreasing right ascension, increasing declination, and line-of-sight direction centered at the optical center of the LMC bar, where $X, Y$, and $Z$ are in units ${ }^{12}$ of kiloparsecs. They approximate the average separation between source and lens as

$\Delta(X, Y)=\frac{I_{b}^{2} \Delta_{b}+I_{d}^{2} \Delta_{d}+I_{b} I_{d} \max \left(\Delta_{b}+\Delta_{d}, \Delta_{b d}\right)}{\left(I_{b}+I_{d}\right)^{2}}$,

where $I_{d}$ and $I_{b}$ are the star count density of the disk and bar, respectively. The line-of-sight depth of the bar and disk are denoted as $\Delta_{b}$ and $\Delta_{d}$, respectively, and $\Delta_{b d}$ is the line-of-sight separation between the midplanes of the bar and disk. In the limit in which the source and the lens are at roughly the same distance, the optical depth can then be expressed as

$$
\tau(X, Y) \sim 10^{-7} \frac{\Sigma(X, Y)}{160 M_{\odot} \mathrm{pc}^{-2}} \frac{\Delta(X, Y)}{1 \mathrm{kpc}} .
$$

The value of $\tau$ depends mainly on two parameters: the displacement $Z_{0}$ between the disk and the bar and the mass of the bar defined by the mass fraction $f_{b}$, which are implicit in the average separation and surface brightness.

${ }^{12}$ For the LMC, $1 \mathrm{kpc}$ is roughly $1^{\circ}$.
In $\S 6$ we vary these parameters to estimate the spatially varying optical depth for different model realizations, and we denote this set of models as model set A.

\subsubsection{Modified Zhao \& Evans (2000) Model Set B}

The optical depth range for self-lensing found by Zhao \& Evans (2000) is significantly larger than the ones found by other studies (e.g., Alcock et al. 1997; Gyuk et al. 2000; Jetzer et al. 2002). As an alternative to model set A, we modify the original Zhao \& Evans (2000) models by improving the effective separation $\Delta^{\prime}(X, Y)$ (S. Nikolaev 2005, private communication), which significantly decreases the optical depth:

$$
\Delta^{\prime}(X, Y)=\frac{I_{b}^{2} \Delta_{b} / 6+I_{d}^{2} \Delta_{d} / 6+I_{b} I_{d} \Psi\left(\Delta_{b}+\Delta_{d}, \Delta_{b d}\right)}{\left(I_{b}+I_{d}\right)^{2}},
$$

where the function $\Psi$ is

$$
\begin{aligned}
& \Psi\left(\Delta_{b}+\Delta_{d}, \Delta_{b d}\right) \\
& = \begin{cases}\Delta_{b d} & \Delta_{b d}>\frac{\Delta_{b}+\Delta_{d}}{2}, \\
\Delta_{b d}+\frac{\left[\left(\Delta_{b}+\Delta_{d}\right) / 2-\Delta_{b d}\right]^{3}}{3 \Delta_{b} \Delta_{d}} & \Delta_{b d} \leq \frac{\Delta_{b}+\Delta_{d}}{2} .\end{cases}
\end{aligned}
$$

We denote this model set as model set B. Note that the first two terms of $\Delta^{\prime}(X, Y)$, the contributions of disk-disk and bar-bar self-lensing, are smaller by a factor of 6 compared to the equivalent expression in the Zhao \& Evans (2000) calculations (see eq. [12]). This takes geometrical considerations into account since source and lens population are the same. The third term is a better approximation for the disk-bar separation: the two cases in equation (15) represent situations (1) where disk and bar are well separated along the line of sight and (2) where they overlap each other. The two limiting cases produce the same result when $\Delta_{b d}=\left(\Delta_{b}+\Delta_{d}\right) / 2$.

It is also important to note that the Zhao models have unrealistically high projected central surface densities, reaching $640 M_{\odot} \mathrm{pc}^{-2}$. A model with parameters as in Gyuk et al. (2000) leads to central densities on the order of $300 \mathrm{M}_{\odot} \mathrm{pc}^{-2}$. The discrepancy is due to the very heavy bar $\left(f_{b}=0.5\right)$ and the quartic bar model (which is more centrally concentrated than a Gaussian bar) used by Zhao \& Evans (2000). Less centralized projected surface densities result in a decrease of the central self-lensing optical depth. As before with model set A, we vary the displacement $Z_{0}$ and the mass fraction $f_{b}$ in $\S 6$ and denote this set of models as model set $\mathrm{B}$.

\section{RESULTS}

In this section, we combine the results from the previous sections in order to obtain a quantitative prediction of the anticipated number of microlensing events. The main observable difference between self-lensing and screen lensing is their distinctive spatial dependence of event rate; therefore, we calculate separately for each field and amplifier the expected number of observed microlensing events using equation (4).

First, we estimate the number of observed stars $N_{\text {obs }}$ for each field and amplifier using equation (5) as described in $\S 5.1$. Figure 5 illustrates this for the example field sme9. The top panel shows the range of LFs we considered. The middle panel shows the detection efficiency, ${ }^{13}$ and the bottom panel shows the

\footnotetext{
13 The efficiency levels out at about $50 \%$ since we integrate in eq. (9) from 0 to only 0.5 , and not to 1 , due to the fact that we do not consider events with amplification smaller than 2 .
} 


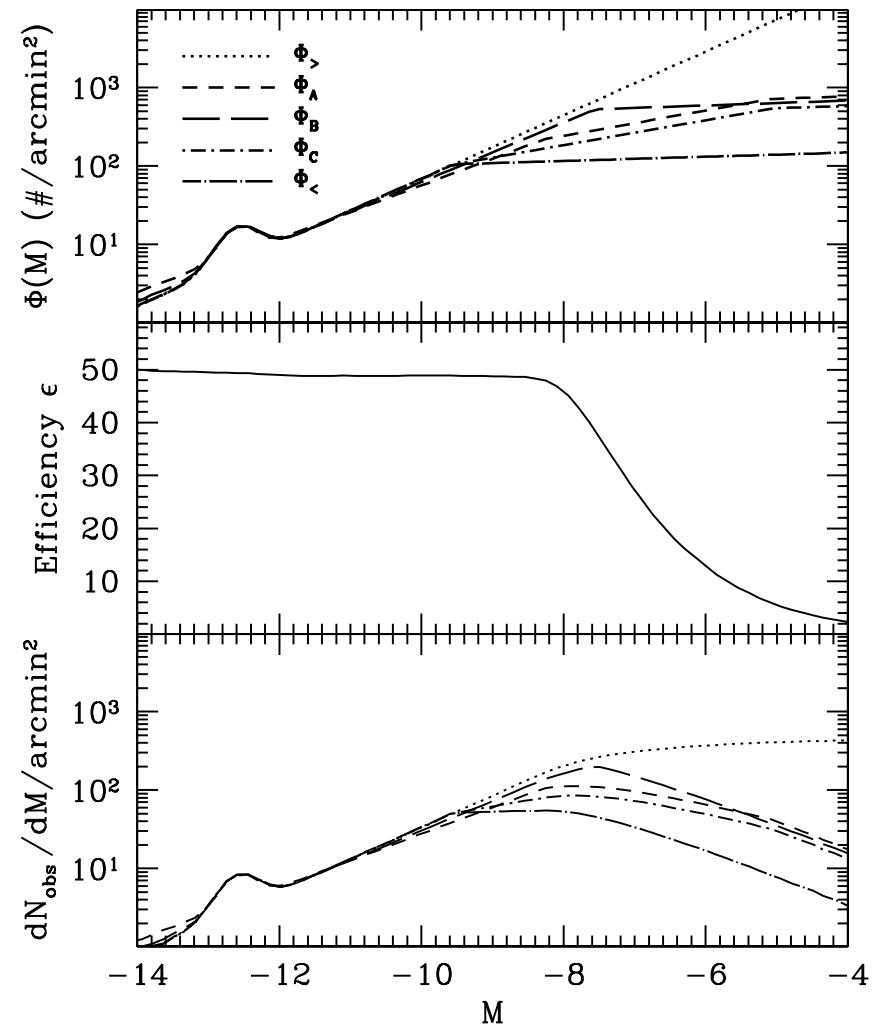

FIG. 5.-Determination of the number of observed stars in field sme9. The top panel shows the LF candidates $\Phi_{>}, \Phi_{A}, \Phi_{B}, \Phi_{C}$, and $\Phi_{<}$, constructed as described in the text. The $x$-axis is the instrumental magnitude. The middle panel shows the event detection efficiency as a function of source-star magnitude. The bottom panel shows the number of monitored stars vs. magnitude, which is the product of the two upper panels. [See the electronic edition of the Journal for a color version of this figure.]

number of useful observed stars per magnitude, which is the product of the two upper panels. Two competing effects, increasing star counts but decreasing efficiency for fainter magnitudes, cause a peak at $M \sim-7.6$. Stars in the magnitude range $-9<M<-6$ will contribute the most to the observed microlensing event rate. Using a zero-point magnitude of 31.3 for this image, this corresponds to a $V R$ filter (unlensed) magnitude range of $22.3<M_{V R}<25.3$. The top panel of Figure 6 shows the sum of the number of observed stars per magnitude bin for all fields and amplifiers, after correcting for exposure times.

We obtain the total number of observed stars by integrating over magnitude (bottom panel). Not surprisingly, the LF chosen does significantly impact the estimated number of observed stars, e.g., choosing $\Phi_{B}$ (short-dashed line) produces $\sim 2$ times more observed stars than $\Phi_{C}$ (short-dash-dotted line). The temporal completeness is taken into account by using $\mathcal{E}_{t} \sim 0.8$ (see $\S 5.2)$.

We can now apply equation (4) to calculate $N_{\mathrm{ml}}$ for any combination of field, amplifier, and optical depth $\tau$. We use $\tau_{\text {screen }}=$ $1.2 \times 10^{-7}$ for the optical depth for screen lensing, as determined by the MACHO Project (Alcock et al. 2000). For self-lensing, we determine the optical depth for the Zhao \& Evans selflensing model sets A and B (see $\S 5.3$ ) using equation (13). The field-dependent $\tau_{\text {self }}$ for each of these models is calculated using each possible combination of $f_{b}=[0.3,0.4,0.5]$ and $Z_{0}=[-1,-0.5,0,0.5,1]$, where $f_{b}$ is the mass fraction of the bar and $Z_{0}$ is the level of displacement between the disk and the bar in kiloparsecs. Based on observations, this covers the likely parameter space of $f_{b}$ and $Z_{0}$. The fields are divided into

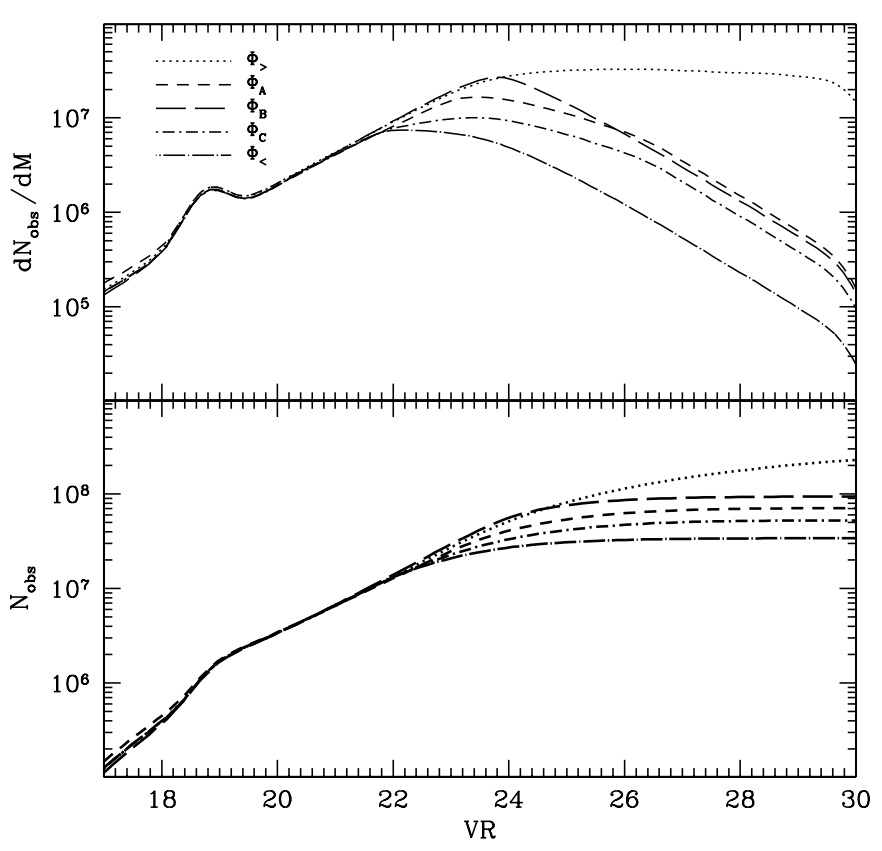

FIG. 6.-Differential and cumulative $N_{\text {obs }}$ for different LFs. The $x$-axis is the apparent $V R$ magnitude, synthesized from standard $V$ and $R$ magnitudes through the equation $V R=A_{0}-2.5 \log \left[A_{1} 10^{-0.4 V}+\left(1-A_{1}\right) 10^{-0.4 R}\right]$, where $A_{0}$ and $A_{1}$ are typically -1.1 and 0.37 , respectively, for MOSAIC II. The top panel shows the number of monitored stars per magnitude bin for all observed fields parameterized by LF. The cumulative number of observed stars is then obtained by integrating over magnitude (bottom panel). The trial LFs $\Phi_{>}, \Phi_{A}, \Phi_{B}, \Phi_{C}$, and $\Phi_{<}$are described in the text. [See the electronic edition of the Journal for a color version of this figure.]

sets $1-5$, based on their respective star density determined with the Zhao \& Evans (2000) LMC bar model (see Fig. 1). Set 1 is the most crowded (yellow) and set 5 is the least crowded (green). For each set of fields, we add up the predicted number of microlensing events for the different models and LFs (see Table 2). The top panel of Figure 7 shows for each set of fields the number of microlensing events for Zhao LMC self-lensing model set A (circles), model set B (squares), and screen lensing (triangles). For clarity the open symbols are plotted with a slight offset for a given set. Note that the spread in the anticipated number of detected microlensing events for screen lensing is solely due to the

TABLE 2

Predicted Microlensing Event Rates for the SuperMaCHO Survey

\begin{tabular}{ccccc}
\hline \hline $\begin{array}{c}\text { Lens Population } \\
(1)\end{array}$ & $\begin{array}{c}\text { Field Set } \\
(2)\end{array}$ & $\begin{array}{c}\text { Minimum } \\
(3)\end{array}$ & $\begin{array}{c}\text { Mean } \\
(4)\end{array}$ & $\begin{array}{c}\text { Maximum } \\
(5)\end{array}$ \\
\hline Screen lensing ..................... & 5 & 4.2 & 5.5 & 6.9 \\
& $4+5$ & 8.2 & 10.8 & 13.8 \\
& All & 25.2 & 34.9 & 45.2 \\
LMC self-lensing: & & & & \\
Model set A ................... & 5 & 0.12 & 0.18 & 0.28 \\
& $4+5$ & 0.47 & 0.87 & 1.6 \\
Model set B ..................... & All & 9.1 & 18.7 & 35.3 \\
& 5 & 0.03 & 0.07 & 0.13 \\
& $4+5$ & 0.25 & 0.53 & 1.13 \\
& All & 5.2 & 10.7 & 21.4 \\
\hline
\end{tabular}

Notes.-Predicted microlensing event totals for a 5 year SuperMACHO survey. Cols. (1) and (2): Lens population and field set. Cols. (3)-(5): Minimum, mean, and maximum event rates for the different models and LFs. The Zhao \& Evans LMC self-lensing model sets A and B differ as described in $\S 5.3$. Note that independent of the overall rate normalization, the ratios of rates are a clear and robust indicator of the nature of the lensing population. 


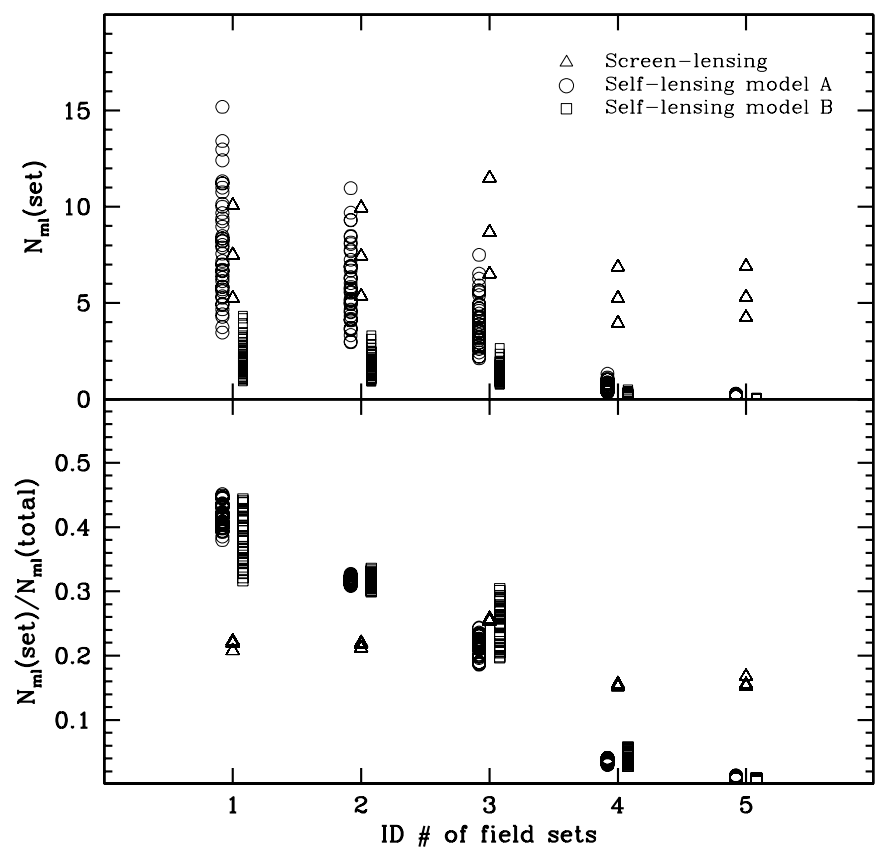

FIG. 7.-Estimated microlensing event counts and differential event rates for the different field sets, as shown in Fig. 1. The triangles indicate screen lensing for the different LFs. The Zhao \& Evans LMC self-lensing model sets A and B are indicated with circles and squares, respectively. The disk-bar displacement of the LMC models varies between -1 and $1 \mathrm{kpc}$, and the bar mass fraction varies between 0.3 and 0.5 . For clarity the open symbols are plotted with a slight offset for a given set. The top panel shows the number of events over a $5 \mathrm{yr}$ survey. The bottom panel shows the differential event rate, normalized to the total number of events detected over the course of the survey. Note that the dependence on the LFs is greatly reduced for the differential rates, as shown in the bottom panel. In particular, by comparing the innermost to the outermost fields we expect to be able to distinguish between the screen-lensing and LMC self-lensing scenarios. [See the electronic edition of the Journal for a color version of this figure.]

different LFs $\left(\Phi_{A}, \Phi_{B}\right.$, and $\left.\Phi_{C}\right)$ used (see Fig. 2). For self-lensing, an additional source of spread is caused by using different values of $f_{b}$ and $Z_{0}$. For sets $1-3$ the intrinsic difference in event rate for self- and screen lensing is of the same order as the systematic errors. The event rate for self-lensing at the center of the bar is particularly large if the displacement between the disk and the bar is large $\left(\left|Z_{0}\right|=1\right)$. For the two outer sets, the rate of selflensing strongly decreases and is well below the event rate for screen lensing.

\section{DISCUSSION}

Generally, the intrinsic difficulty with drawing conclusions from the event rate is that there is a large spread in the predicted rate for a given field due to systematic biases, e.g., the LMC or Galaxy model used and the shape of the LF, especially at the faint end. A way out of this dilemma is to go from an absolute measurement to a relative measurement: instead of considering absolute event rates we investigate the differential event rate for a given field, defined as the ratio of event rate of the field to the total event rate of all fields (see Fig. 7, bottom). Using this normalized quantity to characterize the lensing rate across the LMC suppresses the dependence on LF. This is clearly indicated in comparing the top to the bottom panel in Figure 7. To zeroth order the systematic error arising from LF uncertainty cancels out, and the measurement is much more robust. Table 3 shows the differential event rates for self- and screen lensing.

Despite the anticipated increase in the number of microlensing events for the SuperMACHO survey, we are nevertheless faced
TABLE 3

Predicted Event Rate Ratios

\begin{tabular}{ccccc}
\hline \hline $\begin{array}{c}\text { Lens Population } \\
(1)\end{array}$ & $\begin{array}{c}\text { Field Set } \\
(2)\end{array}$ & $\begin{array}{c}\text { Minimum } \\
(3)\end{array}$ & $\begin{array}{c}\text { Mean } \\
(4)\end{array}$ & $\begin{array}{c}\text { Maximum } \\
(5)\end{array}$ \\
\hline Screen lensing ............... & 5 & $0.15^{*}$ & 0.16 & 0.17 \\
& $4+5$ & $0.30^{*}$ & 0.31 & 0.32 \\
LMC self-lensing: & & & & \\
Model set A .............. & 5 & 0.008 & 0.010 & $0.014^{*}$ \\
& $4+5$ & 0.038 & 0.047 & $0.055^{*}$ \\
Model set B ............... & 5 & 0.004 & 0.007 & $0.011^{*}$ \\
& $4+5$ & 0.032 & 0.051 & $0.070^{*}$ \\
\hline
\end{tabular}

Notes.-Predicted differential microlensing event rates for screen lensing and self-lensing. Cols. (1) and (2): Lens population and field set. Cols. (3)-(5): Minimum, mean, and maximum differential rates for the different models and LFs. In order to be conservative, we use the minimum values for screen lensing and the maximum values for self-lensing, indicated with asterisks. The Zhao \& Evans LMC self-lensing model sets A and B differ as described in $\S$ 5.3. Note that independent of the overall rate normalization, the differential rates are a clear and robust indicator of the nature of the lensing population.

with using small-number statistics to try to distinguish between models for the lensing population. The basic approach we use is to consider what underlying rate could be statistically consistent with a given observed number of events.

We represent these results in confidence level plots, for which we use the statistics given in Gehrels (1986). Because the differences between self- and screen lensing are most pronounced in the outer field sets, we will investigate field set 5 and then the combined field sets 4 and 5 .

Figure 8 is an illustration of the outcome of this process. Assuming that a total of 30 events are detected in the survey (our estimate of the lower bound that we are likely to see for screen lensing), the $x$-axis corresponds to possible observed differential event rates in field set 5. The $y$-axis indicates the lowest allowable actual underlying differential rate, given Poisson statistics. The contours show the 90\% (dot-dashed line), 99\% (curved dashed line), and 99.5\% (solid line) confidence limits on the minimum actual underlying rate, given some measurement on the $x$-axis. The gray area shows the region excluded at $99.5 \%$ confidence.

How can we use this plot? As a Gedankenexperiment, let us assume that screen lensing is indeed the underlying mechanism. In that case, the expectation value for the field set 5 differential event rate is 0.15 for the least favorable LF (see col. [3] in Table 3). Any particular experiment will measure a rate different than exactly 0.15 , but it will most likely realize a value somewhere between 0.1 and 0.2 . As a guide, this screen-lensing expectation value is indicated with a dotted line in Figure 8. The question is, can these measurements exclude self-lensing? The upper limit of the differential rate of all Zhao self-lensing models in set A is 0.014 (see col. [5] in Table 3 and the long-dashed line in the confidence plot). We can exclude these models with $99 \%$ and $99.5 \%$ confidence if we observe a differential rate larger than 0.11 and 0.13 , respectively. Similarly for model set B, the maximum differential rate is 0.011 (straight short-dashed line), and thus it can be excluded at $99.5 \%$ confidence if the observed differential event rate is bigger than 0.12. If SuperMACHO measures a field set 5 differential rate larger than 0.13 , than we can exclude self-lensing as the dominant mechanism at the $99.5 \%$ confidence level.

Let us consider the other case. If self-lensing is the dominant lensing process, then we expect to find many events in the central fields and at most a couple in the outer fields. Therefore in this case it makes sense to calculate the upper bound of the allowed 


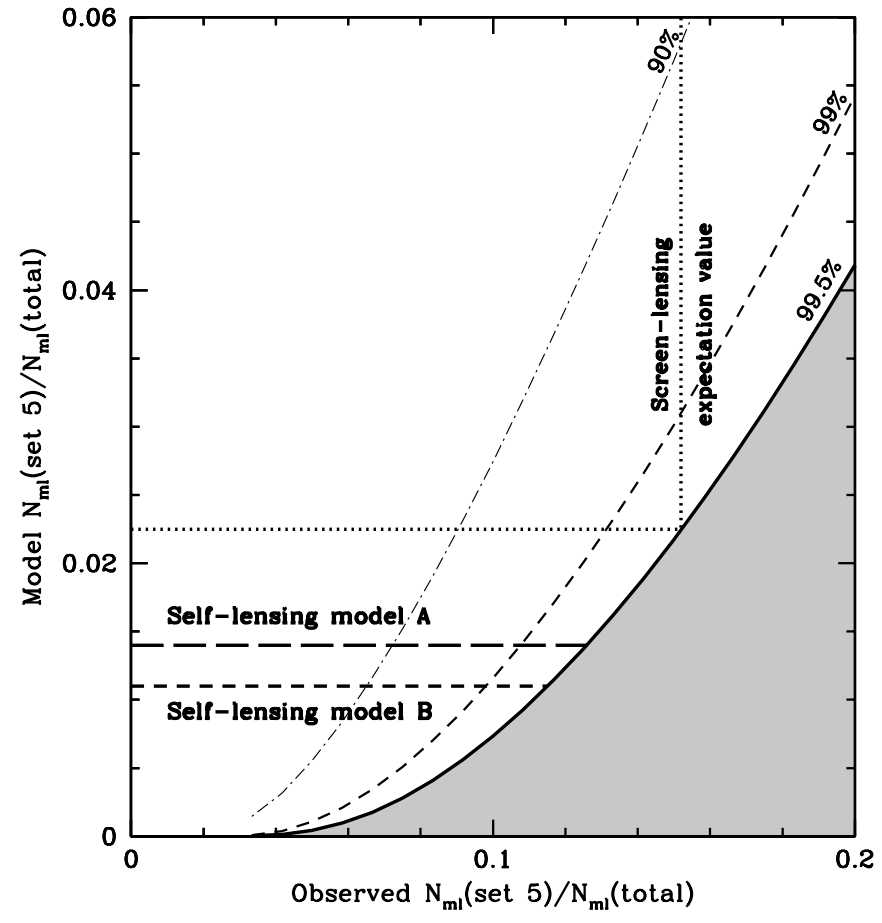

FIG. 8.-Model exclusion plots for any observed differential microlensing event rate. The $x$-axis shows potential observed differential event rates for SuperMACHO field set 5. The $y$-axis corresponds to the allowed underlying event rate that SuperMACHO's observations will use to constrain models. The upper limits to rates allowed by the observations at the $90 \%, 99 \%$, and $99.5 \%$ confidence levels are indicated with dot-dashed, dashed, and solid lines, respectively, based on Poisson statistics assuming a total of 30 detected events. For a given observed differential rate (as plotted on the $x$-axis), the vertical projection of this measurement in the figure will intersect the various confidence contours. The horizontal projection of this intersection to the $y$-axis yields the maximum differential ratio allowed by the observations. In particular, if the prior expectation value for screen lensing of 0.15 is actually observed by SuperMACHO, we can exclude with $99.5 \%$ confidence any model that predicts a ratio of 0.023 or less. This includes the self-lensing models A $(0.014)$ and B (0.011), as described in the text. [See the electronic edition of the Journal for a color version of this figure.]

underlying differential rate given the observed number of events in the outer fields and in total.

For example, the most optimistic self-lensing model A predicts 0.28 events in field set 5 in 5 years. Most likely we will find either 0 or 1 event in this field set (assuming self-lensing only). If we find more than 18 events in total, none of which are in field set 5, then we can exclude screen lensing (assuming a differential rate of 0.17 ; see Fig. 9 , top panel, dotted line) at the $90 \%$ confidence level. Note that our self-lensing models predict a wide range of event rates from 5 to 35 events total during the 5 year survey (see Table 2); thus we can only expect to find that many self-lensing events if the LMC is similar to the models with strong central self-lensing. We would need 42 events to exclude screen lensing at the $99.5 \%$ level and none in field set 5 .

If of 18 or more total events we observe none in field sets 4 and 5 (where screen lensing has an expectation rate of 0.30 ; see Fig. 9 , middle panel, dotted line), we can exclude screen lensing with a confidence level greater than $99.5 \%$. Finding no event is not unlikely since for the combined field set 4 and 5, the event rates predicted from our self-lensing models are between 0.25 and 1.6 events during the 5 years (see Table 2). Finding even one event in field sets 4 and 5 will weaken these conclusions, which can be seen by comparing the exclusion regions of the middle panel to those of the bottom panel.

In reality, the microlensing is probably not caused by a single lens population, but rather by a mixture of several populations.

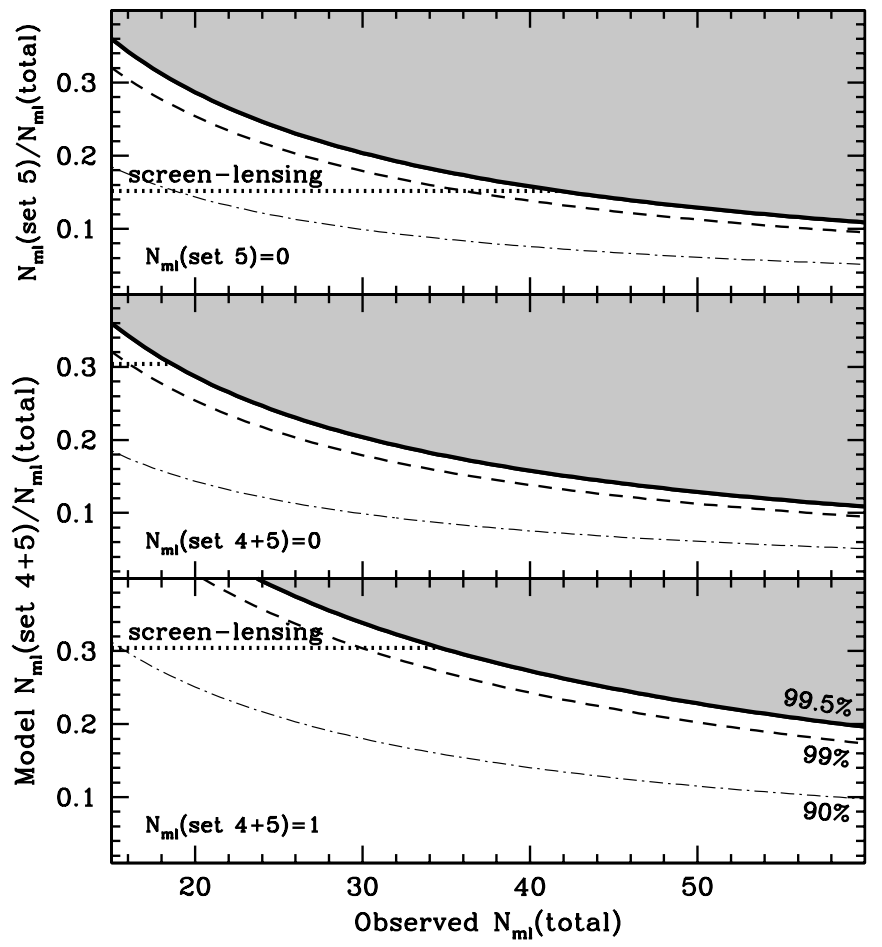

FIG. 9.- SuperMACHO's model discrimination ability as a function of the total number of detected microlensing events. The three panels correspond to different possible experimental outcomes ( 0 or 1 events in the outermost fields). The $x$-axis shows the total number of detected events. The $y$-axis shows the true differential event rate in fields $4+5$ and in field 5 only. The lower boundary of the areas allowed by the observations at the $90 \%, 99 \%$, and $99.5 \%$ confidence levels are indicated with the dotted, dashed, and solid lines, respectively. For a given observed total number of microlensing events ( $x$-axis) and the conditions listed inside each figure window, the confidence contours show the maximum underlying differential rate allowed by the observations. For example, in the case where no event is observed in field sets 4 and 5 (middle panel), if 18 total events are observed, any model predicting an underlying differential event rate (set 4+5) greater than 0.3 (e.g., screen lensing; dotted line) is excluded at $99.5 \%$ confidence. [See the electronic edition of the Journal for a color version of this figure.]

We can expect that it will be more difficult to differentiate between the populations. Still, very recent work reinvestigating MACHO and EROS-2 events finds that even though some of the events are due to self-lensing, the total event rate and spatial distribution cannot be explained by self-lensing alone (Jetzer et al. 2002). Also, a large spectroscopic survey targeting kinematic outliers in the LMC did not find evidence for a significant additional, kinematically distinct population in the LMC (Zhao et al. 2003). The lack of such a population constrains the optical depth of self-lensing to values too small to explain the observed event rate. Even if screen lensing is the cause for only a fraction of the observed event rate, we will be able to detect enough events in the outer field sets to exclude self-lensing as the sole lensing mechanism toward the LMC. If self-lensing is excluded as the sole lensing mechanism, the SuperMACHO event rate will provide a lower limit on the number of MACHOs in the halo of the Milky Way and thus provide a lower limit on their contribution to the Milky Way's dark matter.

\section{SUMMARY AND CONCLUSIONS}

The reported microlensing event rate toward the LMC exceeds that expected from known visible components of our Galaxy, and the source of this observed excess rate is still the subject of discussion. Determining the nature of the lens population will have a great impact on our understanding of Galactic 
and LMC structure and possibly on the nature of dark matter. Possible explanations for the observed lensing can be broadly categorized into screen-lensing and self-lensing scenarios. Using the first-year data of the SuperMACHO Project, we performed completeness analysis by adding artificial stars to the images and estimated the spatial and temporal efficiency of detecting microlensing events in our data sets. We predicted a lower limit of observable microlensing events for both categories using the efficiency in combination with the stellar luminosity functions. We find that the SuperMACHO Project should be able to distinguish between the two categories using the spatial differences in optical depth. Utilizing the differential event rate instead of the event rate itself decreases the impact of systematic errors, rendering the results and conclusions more robust.

The SuperMACHO survey is being undertaken under the auspices of the NOAO Survey Program. We are very grateful for the support provided to the survey program from the NOAO and the National Science Foundation. We are particularly indebted to the scientists and staff at the Cerro Tololo Inter-American Observatory for their assistance in helping us carry out the SuperMACHO survey. We also appreciate the invaluable help of Chance Reschke in building and maintaining the computing cluster we use for image analysis. This project works closely with members of the ESSENCE supernova survey, and we are grateful for their input and assistance.

The support of the McDonnell Foundation, through a Centennial Fellowship awarded to C. Stubbs, has been essential to the SuperMACHO survey. We are most grateful for the Foundation's support for this project. Stubbs is also grateful for support from Harvard University.

K. H. C.'s, S. N.'s, and G. P.'s work was performed under the auspices of the US Department of Energy, National Nuclear Security Administration, at the University of California, Lawrence Livermore National Laboratory, under contract W-7405-Eng-48.

D. L. W. acknowledges financial support in the form of a Discovery Grant from the Natural Sciences and Engineering Research Council of Canada (NSERC).
Alard, C. 2000, A\&AS, 144, 363

Alard, C., \& Lupton, R. H. 1998, ApJ, 503, 325

Alcock, C., et al. 1997, ApJ, 486, 697

1998, ApJ, 499, L9

1999a, ApJS, 124, 171

1999b, PASP, 111, 1539

2000, ApJ, 542, 281

2001a, ApJ, 552, 582

2001b, ApJS, 136, 439

Aubourg, É., Palanque-Delabrouille, N., Salati, P., Spiro, M., \& Taillet, R. 1999, A\&A, 347, 850

Becker, A. C., et al. 2004, ApJ, 611, 418

Bennett, D. P. 2005, ApJ, 633, 906

Bennett, D. P., Becker, A. C., \& Tomaney, A. 2005, ApJ, 631, 301

Calchi Novati, S., et al. 2005, A\&A, in press (astro-ph/0504188)

Chabrier, G. 1999, ApJ, 513, L103 2001, ApJ, 554, 1274

Chabrier, G., Segretain, L., \& Méra, D. 1996, ApJ, 468, L21

Crézé, M., Mohan, V., Robin, A. C., Reylé, C., McCracken, H. J., Cuillandre, J.-C., Le Fèvre, O., \& Mellier, Y. 2004, A\&A, 426, 65

Crotts, A. C. S., Uglesich, R., Gyuk, G., \& Tomaney, A. B. 1999, in ASP Conf. Ser. 182, Galaxy Dynamics, ed. D. R. Merritt, M. Valluri, \& J. A. Sellwood (San Francisco: ASP), 409

Delfosse, X., Forveille, T., Ségransan, D., Beuzit, J.-L., Udry, S., Perrier, C., \& Mayor, M. 2000, A\&A, 364, 217

Evans, N. W., \& Belokurov, V. 2004, preprint (astro-ph/0411222)

Fields, B. D., Freese, K., \& Graff, D. S. 2000, ApJ, 534, 265

Gates, E. I., \& Gyuk, G. 2001, ApJ, 547, 786

Gates, E. I., Gyuk, G., Holder, G. P., \& Turner, M. S. 1998, ApJ, 500, L145

Gehrels, N. 1986, ApJ, 303, 336

Gössl, C. A., \& Riffeser, A. 2002, A\&A, 381, 1095

Gould, A. 1995, ApJ, 441, 77 1999, ApJ, 517, 719

Graff, D. S., Gould, A. P., Suntzeff, N. B., Schommer, R. A., \& Hardy, E. 2000, ApJ, 540, 211

Gyuk, G., Dalal, N., \& Griest, K. 2000, ApJ, 535, 90

Gyuk, G., \& Gates, E. 1999, MNRAS, 304, 281

Holtzman, J. A., et al. 1997, AJ, 113, 656

Hunter, D. A., Light, R. M., Holtzman, J. A., Lynds, R., O’Neil, E. J., \& Grillmair, C. J. 1997, ApJ, 478, 124

Ibata, R. A., Gilmore, G., \& Irwin, M. J. 1995, MNRAS, 277, 781

Ibata, R. A., Irwin, M., Bienaymé, O., Scholz, R., \& Guibert, J. 2000, ApJ, 532, L41

Ibata, R. A., Lewis, G. F., Irwin, M., Totten, E., \& Quinn, T. 2001, ApJ, 551, 294

Ibata, R. A., Richer, H. B., Gilliland, R. L., \& Scott, D. 1999, ApJ, 524, L95 Ivezić, Ž., et al. 2000, AJ, 120, 963

Jetzer, P., Mancini, L., \& Scarpetta, G. 2002, A\&A, 393, 129

\section{REFERENCES}

Jetzer, P., Milsztajn, A., \& Tisserand, P. 2005, in IAU Symp. 225, Gravitational Lensing Impact on Cosmology, ed. Y. Mellier \& G. Meylan (Cambridge: Cambridge Univ. Press), 339

Kilic, M., von Hippel, T., Mendez, R. A., \& Winget, D. E. 2004, ApJ, 609, 766 Kroupa, P., Tout, C. A., \& Gilmore, G. 1993, MNRAS, 262, 545

Mancini, L., Calchi Novati, S., Jetzer, P., \& Scarpetta, G. 2004, A\&A, 427, 61 Méndez, R. A., \& Minniti, D. 2000, ApJ, 529, 911

Minniti, D., Borissova, J., Rejkuba, M., Alves, D. R., Cook, K. H., \& Freeman, K. C. 2003, Science, 301, 1508

Nelson, C. A., Cook, K. H., Axelrod, T. S., Mould, J. R., \& Alcock, C. 2002, ApJ, 573, 644

Newberg, H. J., et al. 2002, ApJ, 569, 245

Olsen, K. A. G. 1999, AJ, 117, 2244

Oppenheimer, B. R., Hambly, N. C., Digby, A. P., Hodgkin, S. T., \& Saumon, D. 2001, Science, 292, 698

Paczyński, B. 1986, ApJ, 304, 1

Phillips, A. C., \& Davis, L. E. 1995, in ASP Conf. Ser. 77, Astronomical Data Analysis Software and Systems IV, ed. R. A. Shaw, H. E. Payne, \& J. J. E. Hayes (San Francisco: ASP), 297

Reid, I. N., Gizis, J. E., \& Hawley, S. L. 2002, AJ, 124, 2721

Reid, I. N., \& Hawley, S. L. 2000, New Light on Dark Stars: Red Dwarfs, LowMass Stars, Brown Dwarfs (New York: Springer)

Reid, I. N., Sahu, K. C., \& Hawley, S. L. 2001, ApJ, 559, 942

Richer, H. B. 2003, in The Dark Universe: Matter, Energy, and Gravity, ed. M. Livio (Cambridge: Cambridge Univ. Press), 24

Sahu, K. C. 1994, Nature, 370, 275

Schechter, P. L., Mateo, M., \& Saha, A. 1993, PASP, 105, 1342

Smith, C. R., Rest, A., Hiriart, R., Becker, A., Stubbs, C., Valdes, F., \& Suntzeff, N. 2002, Proc. SPIE, 4836, 395

Stubbs, C. W. 1999, in ASP Conf. Ser. 165, The Third Stromlo Symposium: The Galactic Halo, ed. B. K. Gibson, T. S. Axelrod, \& M. E. Putman (San Francisco: ASP), 503

Tisserand, P., \& Milsztajn, A. 2005, preprint (astro-ph/0501584)

Udalski, A., Kubiak, M., \& Szymański, M. 1997, Acta Astron., 47, 319

Uglesich, R. R., Crotts, A. P. S., Baltz, E. A., de Jong, J., Boyle, R. P., \& Corbally, C. J. 2004, ApJ, 612, 877

van der Marel, R. P. 2001, AJ, 122, 1827

van der Marel, R. P., \& Cioni, M. L. 2001, AJ, 122, 1807

Vivas, A. K., et al. 2001, ApJ, 554, L33

Weinberg, M. D., \& Nikolaev, S. 2001, ApJ, 548, 712

Woźniak, P. R. 2000, BAAS, 32, 1512

Wu, X. 1994, ApJ, 435, 66

Yanny, B., et al. 2000, ApJ, 540, 825

Zaritsky, D., \& Lin, D. N. C. 1997, AJ, 114, 2545

Zhao, H. 1998, MNRAS, 294, 139

Zhao, H., \& Evans, N. W. 2000, ApJ, 545, L35

Zhao, H., Graff, D. S., \& Guhathakurta, P. 2000, ApJ, 532, L37

Zhao, H., Ibata, R. A., Lewis, G. F., \& Irwin, M. J. 2003, MNRAS, 339, 701 